\title{
Modulation of the Chlamydia trachomatis In vitro transcriptome response by the sex hormones estradiol and progesterone
}

\author{
Ashkan Amirshahi', Charles Wan ${ }^{1}$, Kenneth Beagley ${ }^{1}$, Joanna Latter $^{2}$, lan Symonds ${ }^{2}$ and Peter Timms ${ }^{\text {1* }}$
}

\begin{abstract}
Background: Chlamydia trachomatis is a major cause of sexually transmitted disease in humans. Previous studies in both humans and animal models of chlamydial genital tract infection have suggested that the hormonal status of the genital tract epithelium at the time of exposure can influence the outcome of the chlamydial infection. We performed a whole genome transcriptional profiling study of $C$. trachomatis infection in ECC-1 cells under progesterone or estradiol treatment.

Results: Both hormone treatments caused a significant shift in the sub-set of genes expressed (25\% of the transcriptome altered by more than 2-fold). Overall, estradiol treatment resulted in the down-regulation of 151 genes, including those associated with lipid and nucleotide metabolism. Of particular interest was the upregulation in estradiol-supplemented cultures of six genes ( $O m c B, \operatorname{trp} B, c y d A, c y d B$, pyk and $y g g V$ ), which suggest a stress response similar to that reported previously in other models of chlamydial persistence. We also observed morphological changes consistent with a persistence response. By comparison, progesterone supplementation resulted in a general up-regulation of an energy utilising response.

Conclusion: Our data shows for the first time, that the treatment of chlamydial host cells with key reproductive hormones such as progesterone and estradiol, results in significantly altered chlamydial gene expression profiles. It is likely that these chlamydial expression patterns are survival responses, evolved by the pathogen to enable it to overcome the host's innate immune response. The induction of chlamydial persistence is probably a key component of this survival response.
\end{abstract}

\section{Background}

Chlamydia trachomatis is an obligate intracellular bacterial pathogen that infects the genital and ocular mucosa of humans causing sexually transmitted disease and trachoma respectively. In 2010 the World Health Organization reported 140 million cases of C. trachomatis infections occurred worldwide [1]. In females, C. trachomatis is a common cause of cervicitis, urethritis, with sequelea including ectopic pregnancy, pelvic inflammatory disease, tubal factor infertility, proctitis and chronic pelvic pain. In males, C. trachomatis infections can lead to urethritis, epididymitis and orchitis and it may also contribute to male infertility by directly

\footnotetext{
* Correspondence: p.timms@qut.edu.au

'Institute of Health and Biomedical Innovation, Queensland University of

Technology, Kelvin Grove, Queensland 4059, Australia

Full list of author information is available at the end of the article
}

damaging the sperm [2]. Since approximately $75 \%$ of C. trachomatis infections in women are asymptomatic, research efforts are mainly focused on females $[1,3]$.

Studies using animal models of genital tract Chlamydia infection suggest that the hormonal status of the genital tract epithelium at the time of exposure may influence the outcome of infection. For example, in the commonly used mouse model involving C. muridarum infection, pre-exposed of animals with progesterone is required to achieve infection of $100 \%$ of the animals $[4,5]$. Conversely, guinea pigs are more susceptible to infection following pre-treatment with estradiol [6]. Using a rat model, Kaushic et al. $[7,8]$ found that in rats infected at either estrus or diestrus, without progesterone pre-treatment, no chlamydial inclusions were observed in either the uterus or vagina. In an in vitro model of infection of HeLa cells with C. trachomatis,
C Biomed Central

(c) 2011 Amirshahi et al; licensee BioMed Central Ltd. This is an Open Access article distributed under the terms of the Creative Commons Attribution License (http://creativecommons.org/licenses/by/2.0), which permits unrestricted use, distribution, and reproduction in any medium, provided the original work is properly cited. 
estradiol pre-exposed of cells enhanced both the adherence of chlamydial elementary bodies to the cells as well as the development of chlamydial inclusions [9]. Oral contraceptive use also increases the risk of contracting chlamydial infections compared to women not using contraception [10]. Collectively, these data show that the outcome of chlamydial infection is determined in part by the hormonal status of the epithelium at the time of exposure.

In many cases, chlamydial diseases are associated with a long term or chronic infectious state. In most cases it is difficult to establish whether chronic or recurrent infections arise through the inability of the host to resolve the initial infection or the occurrence of repeated infections with similar species or genotypes. Despite the unresolved nature of the disease etiology, persistence models of chlamydial infection have been studied to provide insight into the nature of chronic disease. Chlamydial persistence is defined as a long-term association between Chlamydia and their host cell in which these organisms remain in a viable but culture-negative state $[11,12]$. Chlamydial persistence is thought to be due in part to a failure to undergo secondary differentiation from RB to EB. Molecular consequences include a 'blockage' in development involving down-regulation of late gene products in persistent infections [13]. The in vitro persistence systems often share altered chlamydial growth characteristics, for example, many studies have described enlarged, and pleomorphic RBs that neither undergo binary fission, nor differentiate back to EBs, but nevertheless continue to replicate their chromosomes. Persistent in vitro infections have been induced by penicillin treatment, amino acid starvation, iron deficiency, Interferon-gamma (IFN- $\gamma$ ) exposure, monocyte infection, phage infection and continuous culture [12-14]. However, a persistence phenotype has not previously been reported to occur in response to altered levels of sex hormones.

Previous data have demonstrated that the metabolic characteristics of persistent chlamydiae were not the same as those of actively growing organisms [12,15-17]. The results reported from Gerard et al. [18] indicated that during the primary phase of active infection, C. trachomatis obtain the energy essential for EB to RB transformation, and also for metabolism, from host cells via ATP/ADP exchange. Through active growth of the RB, the organisms acquire ATP not only from the host, but also via their own glycolytic and pentose phosphate pathways. Gerard et al. (2002) determined that throughout the initial phase of monocyte infection, prior to the complete establishment of persistence, C. trachomatis cells utilized both ATP/ADP exchange and their own pathways to support metabolic needs, even though the overall metabolic rate in the organisms was relatively low. However, when persistence has been established the only source of ATP appears to be the host [18]. This was supported by the finding that, mRNA for glycolytic and pentose phosphate pathway enzymes were absent or severely reduced, suggesting that these systems were partially, if not completely, shut down through persistence. Therefore, C. trachomatis seemed to be merely partial energy parasites on their hosts during active growth, however during persistent infection the organisms appeared to be completely dependent on the host for ATP.

In the current study, we utilised a whole genome microarray to study the changes in chlamydial transcriptional response in in vitro cultured C. trachomatis exposed to either progesterone or estradiol. We found a potentially counter-balancing effect of the two hormones on the chlamydial response.

\section{Methods}

\section{Hormone supplementation of Chlamydia-infected cells}

ECC-1: The ECC-1 is a well-differentiated, steroid responsive human endometrial cell line, which was maintained in phenol red-free $1 \times$ Dulbecco's Modified Eagle Medium/Ham's F12 nutrient mix (DMEM/F12 1:1) (Invitrogen, Carlsbad, CA, USA).

HEp-2: The HEp-2 cell line is a human epithelial cell line, which was maintained in $1 \times$ DMEM containing phenol red, $4.5 \mathrm{~g} / \mathrm{L}$ D-glucose, $110 \mathrm{mg} / \mathrm{L}$ sodium pyruvate, and $584 \mathrm{mg} / \mathrm{L}$ L-glutamine, and supplemented with $500 \mathrm{U} / \mathrm{ml}$ penicillin $\mathrm{G}$ sodium $/ 5,000 \mu \mathrm{g} / \mathrm{ml}$ streptomycin sulphate (Invitrogen).

\section{Hormone preparation}

Lyophilised progesterone and $17 \beta$-estradiol (SigmaAldrich, St. Louis, MO, USA) were solubilised in absolute ethanol to $1 \mathrm{mg} / \mathrm{ml}$ stock. Serum levels of female sex hormones, estradiol and progesterone, fluctuate throughout the menstrual cycle. In this study mean physiological concentrations of $17 \beta$-estradiol $(200 \mathrm{pg} / \mathrm{ml})$ and progesterone $(20 \mathrm{ng} / \mathrm{ml})$, adapted from Williams Textbook of Endocrinology were further diluted using phenol red-free 1× DMEM/F12 medium (Invitrogen), supplemented with $10 \%$ charcoal/dextran-treated FBS (Hyclone). Once the ECC-1 cells had reached 100\% confluence, average physiological concentrations of $17 \beta$ estradiol, progesterone, and a combination of $17 \beta$-estradiol and progesterone (1:1) were added to respective flasks. This hormone exposure was continued throughout the duration of chlamydial infection. Although the physiological concentration of progesterone is higher than estradiol, in this study a combination of 1:1, estradiol and progesterone, was chosen as starting point to merely determine the effect of both hormones together. 
Cells were then incubated for 24 hrs before continuance of experiments.

\section{C. trachomatis serovar D growth and propagation}

C. trachomatis serovar D was grown, maintained and further propagated to create C. trachomatis serovar D stock. C. trachomatis was semi-purified from the infected HEp-2 cells via sonication and vortexing. ECC1 cells were used for $C$. trachomatis serovar D titration. Infected cells were stained utilising the CelLabs Chlamydia Cel LPS staining kit, containing the fluorescein isothiocyanate (FITC)-labelled mouse monoclonal antibody specific for chlamydial lipopolysaccahride (LPS) (CelLabs, Brookvale, Australia), according to manufacturer's instructions.

\section{RNA Extraction}

Total RNA was extracted $48 \mathrm{hrs}$ post infection from infected ECC-1 cells using the Trizol ${ }^{\circledR}$ reagent protocol (Invitrogen) and then treated with DNase. Eukaryotic RNA was removed from total RNA using the Dynabead (poly $\mathrm{A}^{+}$purification kit) (Dynal Biotech ASA, Oslo, Norway) according to manufacturer's instructions and the bacterial mRNA re-suspended in DEPC water. Approximately $2 \mu \mathrm{l}$ of the bacterial mRNA solution was removed to determine the quality and quantity of RNA, using a NanoDrop ${ }^{\circledR}$ Spectrophotometer (NanoDrop Technologies ${ }^{\circledR}$, Wilmington, DE, USA) and associated NanoDrop ND-1000 3.2.1 software (Coleman Technologies Inc., Glen Mills, PA, USA). Extracted RNA was determined to be of high purity, as indicated by the absorbance ratio (A260:A280) being very close to 2.00. The quantity of RNA extracted indicated amplification was not required prior to microarray analysis as the concentration of RNA was sufficient for our experiments.

\section{Whole transcriptome analysis by Affymetrix microarray}

The bacterial mRNA was sent to the AGRF (Australian Genome Research Facility, Melbourne, Australia) for microarray analysis. In vitro RNA transcription was performed to incorporate biotin-labelled ribonucleotides into the cRNA transcripts using the ENZO RNA transcript labelling kit. Labeled cRNAs were purified using the Qiagen kit (according to manufacturer's instructions) and then fragmented to approximately 50 to $200 \mathrm{bp}$ by heating at $94^{\circ} \mathrm{C}$ for $35 \mathrm{~min}$. Fifteen micrograms $(15 \mu \mathrm{g})$ was then hybridized to a Chlamydia whole genome Affymetrix Custom array. The array is an Affymetrix oligonucleotide array format of 1800 features, covering the full C. trachomatis genome ( 875 genes) and containing 8-11 oligonucleotides per target gene, each designed for optimal hybridization to $C$. trachomatis and/or C. pneumoniae and screened for non-specific hybridization against the full human and mouse genomes. After hybridization and subsequent washing using the Affymetrix Fluidics station 400, the bound cRNAs were stained with streptavidin phycoerythrin, and the signal amplified with a fluorescenttagged antibody to streptavidin (Performed by AGRF). Fluorescence was measured using the Affymetrix scanner and the results analysed using GeneChip 1.4 analysis software, resulting in the detection of 1175 genes. A total of 16 chlamydial arrays were analysed with the 4 culture conditions (no hormone, E, P, E+P) $\times$ four replicates. The entire microarray data recorded in Gene Expression Omnibus (GEO) database with accession number GSE24119.

\section{Quantitative RT-PCR}

Quantitative Real-Time PCR was used to validate the microarray data for 20 selected target genes. Each primer pair was used to generate amplicon standards by amplifying previously generated $C$. trachomatis cDNA. cDNA generation was performed using the SuperScript $^{\mathbb{B}}$ III Reverse Transcriptase technique (Invitrogen, Carlsbad, CA, USA). One $\mu$ g of template was added to the PCR mixture containing $0.15 \mu \mathrm{M}$ of gene specific forward and reverse primers, $1 \times$ SYBR Green reaction mastermix, before being made up to a final volume of $25 \mu \mathrm{L}$ with distilled water. The mix is optimized for SYBR Green reactions and contains SYBR Green I dye, AmpliTaq DNA Polymerase, dNTPs and optimized buffer components. Cycling parameters for all reactions were as follows: denaturation at $95^{\circ} \mathrm{C}$ for $10 \mathrm{~min}$; 40 cycles of denaturation at $95^{\circ} \mathrm{C}$ for $15 \mathrm{sec}$ and $1 \mathrm{~min}$ of annealing and extension at $60^{\circ} \mathrm{C}$; and melting curve analysis from $60^{\circ} \mathrm{C}$ to $95^{\circ} \mathrm{C}$. The RotorGene 6000 fast real-time PCR system (Corbett) was used for relative quantification of cDNA copies for the 20 selected genes and an internal reference gene (16S rRNA) was used in all experiments. Quantitation was carried out by using a standard curve based on serial dilutions of the amplicon standards covering 6 logs. Real-time PCR templates for each gene of interest included fresh dilutions of the amplicon standards, 8 cDNA samples $(2 \times 4$ samples per experiment $)$ and distilled water as a negative control. All reactions were performed in triplicate. Reaction tube mastermixes were prepared as per the preparation of amplicon standards described above.

Each gene array profile indicated the expression level of each gene under the differing experimental conditions. To identify genes with similar expression profiles mathematical clustering methods were used, with the resulting hierarchy displayed as dendrograms. 16s rRNA was used as an internal control. The use of an internal control was necessary as the number of genes expressed 
under different hormonal conditions varied substantially and no single gene was constitutively expressed. This method of normalization was particularly important in comparing samples grown in charcoal-stripped, hormone-free media to those in hormone-supplemented cultures.

\section{Microarray data accession number}

The entire microarray data recorded in Gene Expression Omnibus (GEO) database with accession number: GSE24119.

\section{Results and discussion}

Whole transcriptome microarray data confirmed by qRTPCR analyses

We used a whole genome Affymetrix microarray approach to measure the transcriptional responses of C. trachomatis grown in ECC-1 cells supplemented with the female sex hormones, estradiol and progesterone. The resultant data was extracted and filtered through Affymetrix's Gene Chip Operating System (GOCS) version 1.4, and processed using the MAS5 algorithm. Candidate lists of genes were further refined by selecting genes with a greater than 2 -fold up/downregulation and a $p$-value of $<0.05$. Replicate data sets were processed individually and then cross-correlated with each other to find statistically significant changes in gene expression. A total of 16 chlamydial arrays were analysed, with the four culture conditions (no hormone, E, P, E+P), enabling us to have four replicates for each test condition. To confirm the accuracy and reliability of our microarray data, we chose 19 genes that were either up or down-regulated by microarray for analysis by quantitative RT-PCR (Table 1). For 17 of these 19 genes there was complete agreement between the microarray results and the qRT-PCR results. In all cases the fold changes measured by qRTPCR were larger than those recorded using the microarray assay. For the two genes that were not consistent between the two methodologies, the microarray method gave a down-regulation of transcription whereas the qRT-PCR method showed no change in the transcriptional response.

Approximately $25 \%$ of the C. trachomatis transcriptome is altered in response to estradiol or progesterone exposure in vitro

A significant percentage (approximately 25\%) of the $C$. trachomatis transcriptome was altered in response to both hormones. Using a 2-fold change as a cut-off, 63 genes $(7 \%)$ were up-regulated in response to estradiol while 151 genes (17\%) were down-regulated (Table 2). A similar percentage (but different subset) of the transcriptome was altered under progesterone exposure, with 85 genes (10\%) being up-regulated and 135 genes (15\%)
Table 1 Comparison of expression folds change obtained by microarray analysis with fold change obtained by qRT-PCR.

\begin{tabular}{lll}
\hline Gene name & Affymetrix fold change & qRT-PCR fold change \\
\hline gseA & 13.30 up & 27.94 up \\
nqr2 & 9.20 up & 17.32 up \\
ytgD & 9.05 up & 14.07 up \\
ydaO & 5.98 up & 12.51 up \\
pdhA & 5.78 up & 17.30 up \\
recA & 4.12 up & 7.92 up \\
IplA 2 & 3.89 up & 7.41 up \\
trpB & 3.80 up & 11.87 up \\
incA & 3.10 up & 18.04 up \\
fli1 & 2.25 up & 6.80 up \\
sdhB & 22.53 Down & 6.8 Down \\
trxB & 31.44 Down & 5.19 Down \\
pyrH & 21.54 Down & No change \\
miaA & 33.91 Down & 11.74 Down \\
cysS & 19.09 Down & 7.03 Down \\
nrdA & 30.06 Down & 5.16 Down \\
pbp3 & 33.53 Down & 9.43 Down \\
ychF & 21.29 Down & No change \\
yggV & 31.84 Down & 12.11 Down \\
\hline
\end{tabular}

being down-regulated. This represents around 25\% of the transcriptome as a whole, being altered by either hormone alone. When the cut-off was set at 3-fold, 18$20 \%$ of the transcriptome was still changed in response to the sex hormones, but this level dropped to $12 \%$ when a 5 -fold cut-off is used. The full microarray dataset is provided in the GEO database.

Estradiol exposure results in the specific down-regulation of lipid and nucleotide metabolism pathways

In the estradiol-exposed cultures, 151 genes were down-regulated more than 2-fold, while 63 genes were

Table 2 Summary of Chlamydia trachomatis up-regulated and down-regulated genes in response to estradiol or progesterone exposure.

\begin{tabular}{|c|c|c|}
\hline & Estradiol & Progesterone \\
\hline & $\begin{array}{l}\text { No. of genes/\% } \\
\text { of genome }\end{array}$ & $\begin{array}{l}\text { No. of genes } / \% \\
\text { of genome }\end{array}$ \\
\hline \multicolumn{3}{|l|}{ Up regulated } \\
\hline A: > 2-fold, change & $63(7 \%)$ & $85(10 \%)$ \\
\hline B: > 3-fold change & $52(6 \%)$ & 77 (9\%) \\
\hline C: $>$ 5-fold change & $22(2.5 \%)$ & $49(5.5 \%)$ \\
\hline \multicolumn{3}{|l|}{ Down regulated } \\
\hline A: > 2-fold, change & $151(17 \%)$ & 135 (15\%) \\
\hline B: > 3-fold change & $138(15.7 \%)$ & 117 (13\%) \\
\hline C: $>$ 5-fold change & $98(11 \%)$ & $81(9 \%)$ \\
\hline
\end{tabular}

(A) 2-fold cut-off, (B) 3-fold cut-off, (C) 5-fold cut-off. 
up-regulated more than 2-fold during the same period. Of these 213 altered genes, more than 52\% were hypothetical proteins, with no known homologues outside the chlamydiae. Even though nearly $30 \%$ of the chlamydial genome is composed of hypothetical genes, the fact that $52 \%$ of these genes altered their expression by more than 2 -fold in response to estradiol exposure suggests that many of the key changes are uniquely associated with Chlamydia. The five top up-regulated genes (ie. showing the largest fold change) included the Nqr2 subunit of Na-translocating NADH-quinone reductase complex (nqr2) [9.26 fold], UDP-N-acetylmuramoylalanine-D-glutamate ligase, putative (murC/ ddlA) [9.31 fold], V-type ATPase, subunit D, putative $(a t p D)$ [10.23 fold], arginine transport system substrate-binding protein (art) [10.96 fold], and putative glycerol-3-phosphate acyltransferase $(p l s X)$ [16.53 fold]. In addition, the five genes that showed the largest down-regulation of mRNA expression profile include cell division protein FtsI ( $p b p 3$ ) [35.54 fold], nucleoside-triphosphatase $(y g g V)$ [31.84 fold], ribonucleosidediphosphate reductase alpha chain ( $n r d A)$ [30.06 fold], GTP-dependent nucleic acid-binding protein $(y c h F)$ [21.29 fold], and succinate dehydrogenase iron-sulfur subunit $(s d h B)$ [18.82 fold].

When the up- and down-regulated genes were input into the KEGG Pathway database http://www.genome. $\mathrm{jp} / \mathrm{kegg} /$ pathway.html, using the known or predicted chlamydial pathway information, some clustering of transcriptional responses was evident (Table 3). Estradiol clearly induced an overall down-regulation of chlamydial fatty acid biosynthesis, with seven genes being down-regulated at least 2-fold ( $a c c B$, fabF, lipA, fabG, $\left.l p l A \_2\right)$. Estradiol also resulted in a marked down-regulation of the genes involved in chlamydial nucleotide (purine and pyrimidine) metabolism ( $a d k, d n a E$, dut, $n r d A$, surE, $y g g V, r p o C, y g f A, d u t)$. In addition, we also observed a more minor down-regulation in cofactor and vitamin metabolism pathways (hemC, hemN-1, yggV and folD).

Taken together, this overall down-regulation of key pathways is suggestive of a persistence phenotype. The normal chlamydial developmental cycle can be altered under stressful conditions, leading to the formation of aberrant bodies (ABs) which are inhibited in their differentiation back to infectious EBs [11]. Molecular consequences include a 'blockage' in development involving down-regulation of late gene products in persistent infections [19].

The $\operatorname{omc} B$ and $\operatorname{trp} B$ genes are currently the most reliable general markers of chlamydial persistence [12-14,20-22]. The down-regulation trends reported in this project, for these genes under estradiol supplement, were consistent with previous data in the microarray study of IFN- $\gamma-$ mediated $C$. trachomatis serovar D persistence [13]. It has previously been shown that $\operatorname{trp} A$ and $\operatorname{trp} B$ are two genes known to be involved in chlamydial persistence $[12,20]$. Hogan et al. [12] showed that the expression patterns of these two genes were mostly up-regulated in chlamydial persistence. While the expression level of $\operatorname{trp} B$ in our experiment indicated a similar up-regulation, the expression levels of $\operatorname{trp} A$ did not change. As an additional strategy, we attempted to identify chlamydial genes involved in ADP/ATP exchange and energy source pathway reactions in the C. trachomatis genome. This analysis revealed six targets which may be involved in chlamydial persistence (a) two genes encoding proteins involved in the glycolysis pathway ( $p y k, y g g V)(\mathrm{b})$, two genes $(c y d A, c y d B)$ encoding proteins involved in the electron transport system, and (c) two genes encoding proteins involved in the production of tryptophan synthase subunits. Previous data have demonstrated that the metabolic characteristics of persistent chlamydiae were not the same as those of actively growing organisms [12,15-17]. The results reported from Gerard et $a l$. [18] indicated that during the primary phase of active infection, C. trachomatis obtains the energy essential for

Table 3 Categorisation of the up- and down-regulated genes into pathways, as per KEGG.

\begin{tabular}{|c|c|c|c|c|c|}
\hline & \multirow[t]{2}{*}{ Total } & \multicolumn{2}{|c|}{ Up-regulated } & \multicolumn{2}{|c|}{ Down-regulated } \\
\hline & & Estradiol & Progesterone & Estradiol & Progesterone \\
\hline Energy metabolism & 14 & 3 & 4 & 6 & 4 \\
\hline Carbohydrate metabolism & 23 & 2 & 9 & 1 & - \\
\hline Lipid metabolism & 27 & 1 & 2 & 7 & 8 \\
\hline Nucleotide metabolism & 29 & - & 1 & 16 & 3 \\
\hline Amino acid metabolism & 30 & 3 & 8 & 3 & 3 \\
\hline Metabolism of other amino acids & 4 & - & - & - & - \\
\hline Metabolism of cofactors and vitamins & 33 & - & 1 & 6 & 3 \\
\hline Glycan biosynthesis and metabolism & 16 & 2 & 6 & 1 & 2 \\
\hline \multirow[t]{2}{*}{ Biosynthesis of secondary metabolism } & 15 & 1 & 1 & 3 & 4 \\
\hline & & 12 & 32 & 43 & 27 \\
\hline
\end{tabular}

The numbers represent the number of pathways (not genes) affected following exposure with either Estradiol or progesterone. 
EB to RB transformation, and also for metabolism, from host cells via ATP/ADP exchange. Through active growth of the RB, the organisms acquire ATP not only from the host, but also via their own glycolytic and pentose phosphate pathways. Gerard et al. (2002) showed that throughout the initial phase of monocyte infection, prior to the complete establishment of persistence, C. trachomatis cells utilized both ATP/ADP exchange and their own pathways to support metabolic needs, even though the overall metabolic rate in the organisms was relatively low. However, when persistence has been established, the only source of ATP seemed to be the host [18]. That is, mRNA for glycolytic and pentose phosphate pathway enzymes were absent or severely reduced, suggesting that these systems were partially, if not completely, shut down during persistence. Therefore, $C$. trachomatis seems to be only partial energy parasites on their hosts during active growth, however during persistent infection, the organisms appear to be completely dependent on the host for ATP.
Most notably in our current project, $p y k$ and $y g g V$ were strongly down-regulated (3-fold and 10-fold respectively) following supplementation with estradiol, which may contribute to a reduction in the rate of glycolysis biosynthesis during persistence. Two other well known chlamydial persistence genes ( $c y d A, c y d B)$, which play a part in the electron transport system were also down-regulated (8-fold and 4-fold respectively) in the presence of estradiol.

The other key persistence-suggestive change was observed at the morphological level. It has been previously reported by several authors $[13,23,24]$ that chlamydiae show abnormal morphology under persistence conditions. We analysed both un-exposed as well as hormone-exposed C. trachomatis infected ECC-1 cell cultures using Transmission Electron Microscope (TEM) analysis (Figure 1). Under normal cell culture conditions (ie cell culture media supplemented with FCS) we observed normal chlamydial inclusion growth

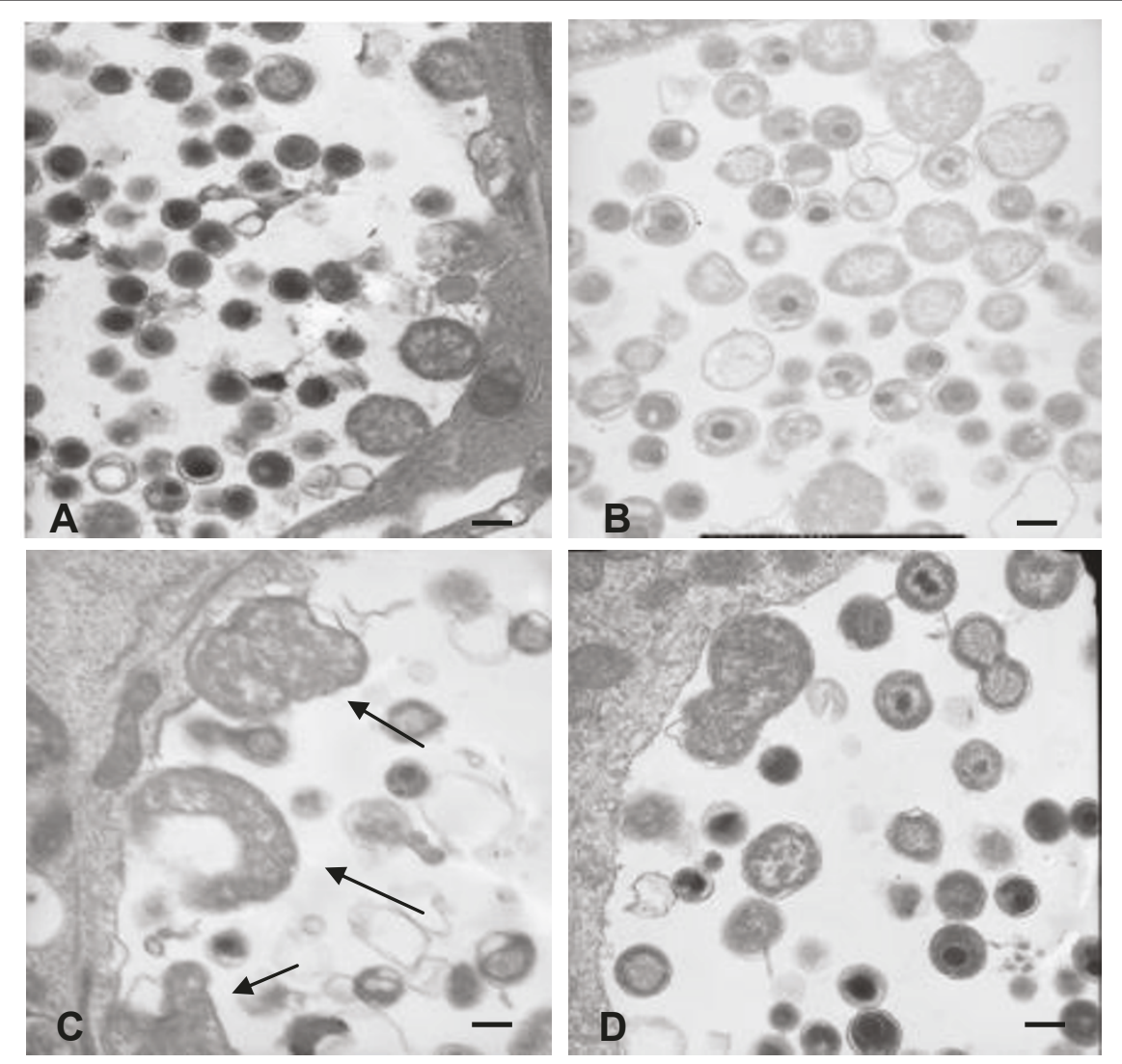

Figure 1 Transmission electron micrographs of $C$. trachomatis inclusion development in ECC-1 cells at 48 hours under different hormonal conditions estradiol and/or progesterone. (A) ECC-1 cells grown in normal FCS supplemented cell culture. Typical RB forms are present at 24 hours post infection (B) No hormone supplemented stripped FCS media. Once again normal RB morphology was observed under this condition; RBs appeared similar to normal FCS supplemented cell culture. (C) Estradiol supplemented, RBs were distinctly different, appearing as large aberrant form. Estradiol supplementation of infected cells, resulting in smaller inclusions containing enlarged, atypical RB forms (arrows). (D) Progesterone supplemented, shape and morphology of RBs were normal including binary fission. Morphological examination of progesterone exposed cultures with TEM did not show any evidence of aberrant, persistent forms. Magnification: $\times 20 \mathrm{~K}$, marker represent $200 \mathrm{~nm}$. 
and development as depicted by a mixture of characteristic RBs and EBs of normal size and shape (Figure 1, Panel A). By comparison, when we grew the chlamydiae in charcoal stripped foetal calf serum (hormone free media), supplemented with estradiol, we observed typical chlamydial persistence inclusions containing aberrant, enlarged RBs which had not differentiated into EBs (Figure 1, Panel C). The morphological features that we observed associated with hormone-mediated persistence demonstrate similarities to those observed by others for persistence induced by IFN- $\gamma$ and penicillin.

\section{Progesterone exposure induces an up-regulated energy utilising chlamydial response}

Overall, 85 chlamydial genes were observed to have twofold or greater up-regulated gene expression levels in the presence of progesterone. The five top genes that were observed with this mRNA expression profile encode for proton or sodium-glutamate symport protein (gltT) [33.4 fold], the putative glycerol-3-phosphate acyltransferase $(p l s X)$ [16.17 fold], glucose inhibited division protein (lplA_2) [11.9 fold], NADH-quinone reductase complex (nqr2) [10.95 fold] and polynucleotide adenylyltransferase (pcnB_1) [10.75 fold]. In addition to these 85 genes, 135 chlamydial genes were observed to have a reduced gene expression profile in response to the presence of progesterone. The five top down regulated genes include exoribonuclease II ( $v a c B)$ [67.96 fold], isopentenylpyrophosphate transferase (miaA) [33.91 fold], cysteinyl-tRNA synthetase (cys $S$ ) [33.64 fold], thioredoxin reductase $(\operatorname{tr} x B)$ [33.44fold], and ribonucleotidediphosphate reductase subunit alpha $(n r d A)$ [29.25 fold]. 103 genes had unknown annotated functions (hypothetical genes).

By comparison to the estradiol response, which resulted in a down-regulation of fatty acid and nucleotide metabolism pathways, progesterone exposure had no or little effect on these pathways but did result in a significant up-regulation of the TCA cycle and glycolysis pathways (Table 3 ). In some aspects the progesterone response was opposite or counter-balancing to the estradiol response. Progesterone resulted in a general up-regulation of carbohydrate metabolism pathways as well as an up-regulation of amino acid metabolism pathways.

The progesterone-mediated response mounted by Chlamydia reflects the host's flux of metabolites. Progesterone has been reported to have a suppressive effect in general on estradiol [25], and after prolonged exposure, it appears that Chlamydia is diverting specific pathways to compensate. From the top five up-regulated genes, three genes are associated with Chlamydia-specific salvage pathways: lplA_2 for regeneration of lipoic acid; and $p l s X$ for the formation of glycerophospolipids and phosphotidylcholine regeneration; $n q r 2$ for the regeneration of $\mathrm{NAD}(\mathrm{P}) \mathrm{H}$ and free-radicals. The association with the top five down-regulated genes appears to align with control at the transcriptional/translational level. For example, the gene encoding miaA and cys $S$ have associated functions with translation, through transfer RNA molecules. nrdA plays an important role in nucleotide regeneration and our observation that expression of this gene was down 29-fold, suggests that one mechanism being employed by $C$. trachomatis is to reduce cellular multiplication.

While these chlamydial transcriptome changes might be a direct result of the effect of the hormones on the chlamydiae it is likely that the major effects are indirect, via the host cells. As an intracellular pathogen, most of the chlamydial response to the hormones is most likely an indirect response to changes in the host cells. In a parallel study (Wan et al., manuscript submitted) we have analysed the host cell response to these hormones and have found a cascade of changes. It is likely therefore that the chlamydial transcriptome changes are in response to these host cell changes. It is known that hormones have a major effect on host cell innate immune pathways. For example, the expression of antimicrobial peptides such as human defensin 5 (HD-5 $[26])$, lactoferrin $[27,28]$, and secretory leukocyte protease inhibitor (SLPI) [29] are all influenced by changes in female sex hormones, as is the recruitment of neutrophils, macrophages and NK cells into the reproductive tract [30]. Furthermore, chlamydial infection of progesterone-exposed endocervical cells results in increased mRNA levels for multiple chemokines, cytokines as well as up-regulation of various interferon pathways in these cells (Wan et al. manuscript submitted) suggesting that the chlamydial changes may be in response to the altered host cell environment. In the present study we analysed the effects of either progesterone or estradiol separately. In reality, both hormones are continually present, but their levels fluctuate during the various stages of the estrous cycle. This hormonal cycling may have the effect of causing the chlamydiae to alternate between cycles of productive growth and cycles of persistence or dormancy. Given the 28 day duration of the human female menstrual cycle and the 2-3 day growth cycle of $C$. trachomatis, such cycling is a real possibility and may be of survival benefit to the chlamydiae.

\section{Conclusions}

This is the first study to demonstrate transcriptional analysis of Chlamydia trachomatis genes under different hormonal conditions. Previous studies provided evidence that the hormonal environment at the time of pathogen exposure can have anclinical effect on the outcome of a microbial infection in the genital tract. In the current experiments, we examined the effect of the hormonal 
environment on (a) C. trachomatis gene expression and (b) the type of inclusions that develop. In our experiments, evidence of long-term persistent chlamydial infections was observed. Collectively our data suggest that hormonal supplementation; estradiol in particular, may directly or indirectly play an important role in the development of chlamydial persistence. The data may help to explain why infections are more common in the estrogen-dominant phase of the menstrual cycle and suggest that estradiol favours the development of persistent infections that may allow Chlamydia to; (a) resist common antibiotic therapy and (b) survive the innate immune response to infection, thereby facilitating repeated reactivation of infection that drives damaging immunopathology.

\section{Acknowledgements}

We would like to thank Dr. Deb Stenzel for technical assistance and advice with TEM; and Dr. Cameron Hurst for statistical advice. This research was supported by funding from the National Health and Medical Research Council (NHMRC grant no. 401245).

\section{Author details}

'Institute of Health and Biomedical Innovation, Queensland University of Technology, Kelvin Grove, Queensland 4059, Australia. ${ }^{2}$ Faculty of Health, The University of Newcastle, Callaghan, New South Wales 2308, Australia.

\section{Authors' contributions}

AA carried out the molecular genetic and microarray studies, participated in the microarray analysis and drafted the manuscript. CW designed microarray chip and participated in the microarray analysis. KB conceived the study and revised the manuscript critically for important intellectual content. JL participated in the cell culture and provided the initial samples. IS revised the manuscript critically for important intellectual content. PT participated in the design of the study, project coordination and helped to draft the manuscript. All authors read and approved the final manuscript.

\section{Competing interests}

The authors declare that they have no competing interests.

Received: 17 March 2011 Accepted: 25 June 2011

Published: 25 June 2011

\section{References}

1. Beagley KW, Timms P: Chlamydia trachomatis infection: incidence, health costs and prospects for vaccine development. J Reprod Immunol 2000, 48(1):47-68.

2. Cunningham AKelly, Beagley KW: Male Genital Tract Chlamydial Infection: Implications for Pathology and Infertility. Biol Reprod 2008, 79(2):180-189.

3. Westrom L, Mardh PA: Chlamydial salpingitis. Br Med Bull 1983, 39(2):145-150.

4. Rank RG: Animal models for urogenital infections. Methods Enzymol 1994, 235:83-93.

5. Berry $\sqcup$, Hickey DK, Skelding KA, Bao S, Rendina AM, Hansbro PM, Gockel CM, Beagley KW: Transcutaneous immunization with combined cholera toxin and $\mathrm{CpG}$ adjuvant protects against Chlamydia muridarum genital tract infection. Infect Immun 2004, 72(2):1019-1028.

6. Rank RG, White HJ, Hough AJ, Pasley JN, Barron AL: Effect of estradiol on chlamydial genital infection of female guinea pigs. Infect Immun 1982, 38(2):699-705

7. Kaushic C, Murdin AD, Underdown BJ, Wira CR: Chlamydia trachomatis infection in the female reproductive tract of the rat: influence of progesterone on infectivity and immune response. Infect Immun 1998, 66(3):893-898.
8. Kaushic C, Zhou F, Murdin AD, Wira CR: Effects of estradiol and progesterone on susceptibility and early immune responses to Chlamydia trachomatis infection in the female reproductive tract. Infect Immun 2000, 68(7):4207-4216.

9. Bose SK, Goswami PC: Enhancement of adherence and growth of Chlamydia trachomatis by estrogen treatment of HeLa cells. Infect Immun 1986, 53(3):646-650.

10. Baeten JM, Nyange PM, Richardson BA, Lavreys L, Chohan B, Martin HL, Mandaliya K, Ndinya-Achola JO, Bwayo JJ, Kreiss JK: Hormonal contraception and risk of sexually transmitted disease acquisition: results from a prospective study. Am J Obstet Gynecol 2001, 185(2):380-385.

11. Abdelrahman YM, Belland RJ: The chlamydial developmental cycle. FEMS Microbiol Rev 2005, 29(5):949-959.

12. Hogan RJ, Mathews SA, Mukhopadhyay S, Summersgill JT, Timms P: Chlamydial persistence: beyond the biphasic paradigm. Infect Immun 2004, 72(4):1843-1855.

13. Belland RJ, Nelson DE, Virok D, Crane DD, Hogan D, Sturdevant D, Beatty WL, Caldwell HD: Transcriptome analysis of chlamydial growth during IFN-gamma-mediated persistence and reactivation. Proc Natl Acad Sci USA 2003, 100(26):15971-15976.

14. Morrison RP: New insights into a persistent problem - chlamydial infections. J Clin Invest 2003, 111(11):1647-1649.

15. Polkinghorne A, Hogan RJ, Vaughan L, Summersgill JT, Timms P: Differential expression of chlamydial signal transduction genes in normal and interferon gamma-induced persistent Chlamydophila pneumoniae infections. Microbes Infect 2006, 8(1):61-72.

16. Jones ML, Gaston JS, Pearce JH: Induction of abnormal Chlamydia trachomatis by exposure to interferon-gamma or amino acid deprivation and comparative antigenic analysis. Microb Pathog 2001, 30(5):299-309.

17. Wyrick PB: Chlamydia trachomatis persistence in vitro: an overview. J Infect Dis 201(Suppl 2):S88-95.

18. Gerard HC, Freise J, Wang Z, Roberts G, Rudy D, Krauss-Opatz B, Kohler L, Zeidler H, Schumacher HR, Whittum-Hudson JA, et al: Chlamydia trachomatis genes whose products are related to energy metabolism are expressed differentially in active vs. persistent infection. Microbes Infect 2002, 4(1):13-22.

19. Belland RJ, Zhong G, Crane DD, Hogan D, Sturdevant D, Sharma J, Beatty WL, Caldwell HD: Genomic transcriptional profiling of the developmental cycle of Chlamydia trachomatis. Proc Natl Acad Sci USA 2003, 100(14):8478-8483.

20. Akers JC, Tan M: Molecular mechanism of tryptophan-dependent transcriptional regulation in Chlamydia trachomatis. J Bacteriol 2006, 188(12):4236-4243.

21. McClarty G, Caldwell HD, Nelson DE: Chlamydial interferon gamma immune evasion influences infection tropism. Curr Opin Microbiol 2007, 10(1):47-51.

22. Nelson DE, Virok DP, Wood H, Roshick C, Johnson RM, Whitmire WM, Crane DD, Steele-Mortimer O, Kari L, McClarty G, et al: Chlamydial IFNgamma immune evasion is linked to host infection tropism. Proc Natl Acad Sci USA 2005, 102(30):10658-10663.

23. Beatty WL, Morrison RP, Byrne Gl: Persistent chlamydiae: from cell culture to a paradigm for chlamydial pathogenesis. Microbiol Rev 1994, 58(4):686-699.

24. Beatty WL, Byrne Gl, Morrison RP: Morphologic and antigenic characterization of interferon gamma-mediated persistent Chlamydia trachomatis infection in vitro. Proc Natl Acad Sci USA 1993, 90(9):3998-4002.

25. Kaushic C, Grant K, Crane M, Wira CR: Infection of polarized primary epithelial cells from rat uterus with Chlamydia trachomatis: cell-cell interaction and cytokine secretion. Am J Reprod Immunol 2000, 44(2):73-79.

26. Quayle AJ, Porter EM, Nussbaum AA, Wang YM, Brabec C, Yip KP, Mok SC: Gene expression, immunolocalization, and secretion of human defensin5 in human female reproductive tract. Am J Pathol 1998, 152(5):1247-1258.

27. Walmer DK, Wrona MA, Hughes CL, Nelson KG: Lactoferrin expression in the mouse reproductive tract during the natural estrous cycle: correlation with circulating estradiol and progesterone. Endocrinology 1992, 131(3):1458-1466. 
28. Cohen MS, Britigan BE, French M, Bean K: Preliminary observations on lactoferrin secretion in human vaginal mucus: variation during the menstrual cycle, evidence of hormonal regulation, and implications for infection with Neisseria gonorrhoeae. Am J Obstet Gynecol 1987, 157(5):1122-1125.

29. Fahey JV, Wira CR: Effect of menstrual status on antibacterial activity and secretory leukocyte protease inhibitor production by human uterine epithelial cells in culture. J Infect Dis 2002, 185(11):1606-1613.

30. Beagley KW, Gockel CM: Regulation of innate and adaptive immunity by the female sex hormones oestradiol and progesterone. FEMS Immunol Med Microbiol 2003, 38(1):13-22.

doi:10.1186/1471-2180-11-150

Cite this article as: Amirshahi et al: Modulation of the Chlamydia trachomatis In vitro transcriptome response by the sex hormones estradiol and progesterone. BMC Microbiology 2011 11:150.

\section{Submit your next manuscript to BioMed Central} and take full advantage of:

- Convenient online submission

- Thorough peer review

- No space constraints or color figure charges

- Immediate publication on acceptance

- Inclusion in PubMed, CAS, Scopus and Google Scholar

- Research which is freely available for redistribution

Submit your manuscript at www.biomedcentral.com/submit 